\title{
Evaluation of Cognitive Decline Using Electroencephalograph Beta/Alpha Ratio During Brain-Computer Interface Tasks
}

\author{
Yuri NISHIZAWA*, Hisaya TANAKA **, Raita FUKASAWA***, Kentaro HIRAO***, \\ Akito TSUGAWA*** and Soichiro SHIMIZU*** \\ * Department of Computer Science, Faculty of Informatics, Kogakuin University, \\ 1-24-2 Nishishinjuku, Shinjuku-ku, Tokyo 163-8677, Japan \\ j117215@ns.kogakuin.ac.jp \\ ** Graduate School of Engineering, Kogakuin University, 1-24-2 Nishishinjuku, Shinjuku-ku, Tokyo 163-8677, Japan \\ hisaya@cc.kogakuin.ac.jp \\ *** Department of Geriatric Medicine, Tokyo Medical University, 6-1-1 Shinjuku, Shinjuku-ku, Tokyol60-8402, Japan
}

\begin{abstract}
Japan is currently witnessing an increase in the number of individuals with dementia. According to a survey, there were 4.62 million people with dementia in Japan in 2012 and more than 7 million in 2025 . The early detection of dementia is crucial to delay the progression of symptoms. As such, in our laboratory, we are developing a dementia screening tool using a character-input-type brain-computer interface (BCI). In this study, the spelling-type-BCI is used to analyze and verify electroencephalograph (EEG) data obtained in the frequency band. We aim to clarify how EEG characteristics differ between healthy subjects and those with mild cognitive impairment (MCI). As a result, we observed that a high possibility exists that there is a difference in the mean value of $\beta / \alpha$ and the generation rate of $\theta$ waves among healthy subjects, patients with MCI, and patients with Alzheimer's dementia. This difference can likely be attributed to our consideration of $\beta / \alpha$ as an index of the degree of concentration associated with cognitive decline and $\theta$ waves as the characteristics of the EEGs of patients with dementia. Based on these results, measuring $\beta / \alpha$ and $\theta$ waves could lead to the early detection and diagnosis of dementia.
\end{abstract}

Keywords: Dementia, $\beta / \alpha, \theta$ wave, electroencephalograph, brain-computer interface

\section{Introduction}

One of the various social problems in Japan is the increasing number of people with dementia. According to a survey by the Ministry of Health, Labor and Welfare, there were 4.62 million dementia patients in Japan in 2012, and it was predicted that there would be more than 7 million in 2025 [1]. Black et al. have quantified the diagnostic pathway from cognitive impairment to dementia for individuals in Japan. It was revealed that $81.2 \%$ of specialists test and examine patients for dementia [2]. This shortage of doctors who perform medical examinations that are designed to detect dementia is also considered a problem because the early detection of dementia can delay the progression of symptoms. Thus, developing methods of early diagnosis is imperative.

In our laboratory, we are using a character-input-type brain-computer interface (BCI) to develop a screening tool to detect and diagnose dementia early on. According to Kurihara et al., a person's cognitive decline can be investigated using a spelling-BCI to measure the erroneous input distance value. However, their methods did not show any significant difference between healthy subjects and those with mild cognitive impairment (MCI), which is necessary for the early diagnosis of dementia. As a result, they concluded that a new index must be introduced [3].

This study aims to introduce a new index into the analysis of the electroencephalograph (EEG) data obtained by our team and identify the difference between healthy subjects and those in the pre-dementia stage. We clarify the difference between the EEGs of healthy subjects and patients with dementia by analyzing the EEG frequencies for introducing a new index. In addition, we compare the results according to the degree of dementia progression to separately analyze these healthy subjects and those in the pre-dementia stage.

\section{Dementia}

Dementia is a condition in which normally developed intelligence is reduced or lost, which in turn interferes with daily life. There are several types of dementia, the 
main ones being Alzheimer's dementia (AD), vascular dementia, and Lewy body dementia. In this study, the condition of subjects is analyzed by dividing them into healthy subjects, those with MCI, and those with AD. MCI refers to a pre-dementia condition that does not interfere with daily life but is not completely normal. By definition, there are complaints of forgetfulness by the person or family, and general cognitive function is within the normal range. The pre-dementia stage described so far refers to this condition. AD is the stage of dementia that interferes with and individual's life due to the degeneration of the brain due to an unknown cause. The main symptom is memory impairment, which impairs the ability to learn new things and remember existing information.

\section{Experiment}

\subsection{Conditions}

Experiments were conducted using the spelling-BCI of Kurihara et al [3]. Characters were input by displaying the grid on the screen (Fig. 1). First, only the target characters were displayed on the screen and presented to the subject. The subjects were instructed to look at the target character, and the grid was then blinked in units of rows and columns. When the row or column containing the target character blinked, the subject stated the character aloud. This was done for each task character, and the five- and six-character tasks were each performed twice. In other words, the EEGs were measured four times. This data was used to analyze the four EEG data for each subject, excluding defects. The setup of the experiment is shown in Fig. 2.

\subsection{Subject information}

This study was conducted on 56 patients in their 60 s and 90s who visited the Tokyo Medical University outpatient department from 2016 to 2020 . Of these 56 patients, 6 were healthy, 25 had MCI, and 25 had AD. The average age was $79.62 \pm 4.90$ years. This experiment was carried out with the approval of the Ethics Research Committee of Tokyo Medical University (early diagnosis of dementia using Brain-Computer Interface (BCI) 2016-083). All subjects provided written informed consent.

\subsection{Electrodes}

In this experiment, electrodes are placed using 8 of the 10-20 electrode placement methods standardized by the International Electroencephalography Society (Fz, Cz, P3, $\mathrm{P} 4, \mathrm{Pz}, \mathrm{Oz}, \mathrm{O} 1$, and $\mathrm{O} 2$ ).
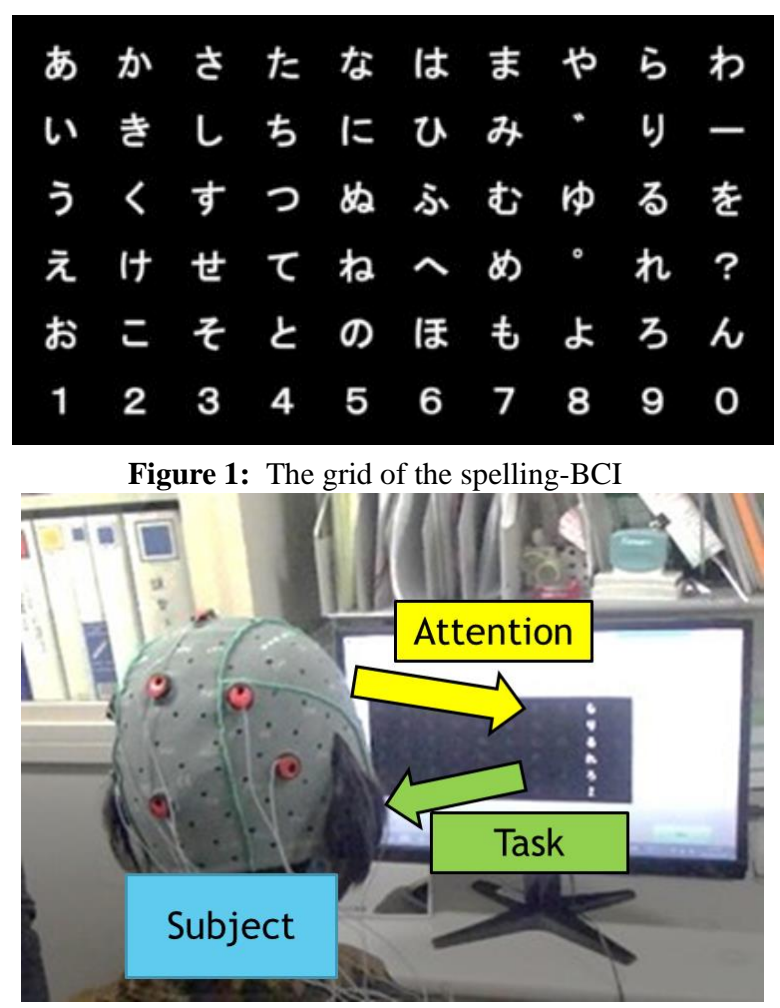

Figure 2: Setup of the experiment

\section{Analysis}

\subsection{EEG bands and indicators}

EEG analysis is regarded as an effective diagnostic tool for dementia, especially AD. A study of EEG readings of patients with dementia by Mori and Otomo revealed that the $\beta / \alpha$ ratio (the ratio of $\beta$ and $\alpha$ waves generated) and dementia are closely related [4]. A study of EEG analysis at the learning time using a simple EEG by Hirai et al. confirmed that the $\beta / \alpha$ ratio increases when mental load is required. Based on this, they reported that the degree of mental load and the degree of concentration can be measured by observing the $\beta / \alpha$ ratio [5]. Haranaka et al. also measured the EEG activity of drivers driving a circuit [6]. They focused on the generation ratio of $\alpha$ and $\beta$ waves and $\beta / \alpha$. In this study, we measured the generation rate of $\alpha, \beta$, and $\theta$ waves. In addition, we analyzed $\beta / \alpha$ as an index of concentration. Each equation is shown below.

$$
\begin{gathered}
\theta \%=\frac{\Sigma \theta}{\Sigma S} \times 100 \\
\alpha \%=\frac{\Sigma \alpha}{\Sigma S} \times 100 \\
\beta \%=\frac{\Sigma \beta}{\Sigma S} \times 100 \\
\frac{\beta}{\alpha}=\frac{\Sigma \beta}{\Sigma \alpha}
\end{gathered}
$$

\subsection{Hypothesis}

In this study, we tested our hypothesis using the $\beta / \alpha$ ratio, as explained in Section 3.1. The average value of 
$\beta / \alpha$ was separately measured for healthy subjects, those with MCI, and those with AD. As a result, we expected $\beta / \alpha$ to decrease as cognitive function declined. This is based on the premise that $\beta / \alpha$ is an index of concentration. Since healthy subjects can concentrate on the task of looking at a character, $\beta$ waves are considered to be stronger than $\alpha$ waves. In comparison, subjects with MCI were expected to be less able to concentrate than healthy subjects due to their symptoms. In addition, the subjects with $\mathrm{AD}$ were considered to have the lowest concentration because they have dementia, and the $\beta / \alpha$ associated with that group is the lowest of the three.

\subsection{Results}

The analysis results of the averaged $\beta / \alpha$ values are shown in Table 1 and Fig. 3. First, Table 1 summarizes the mean values of $\beta / \alpha$ for each of the eight electrodes used. The overall average is calculated for each subject's condition. One-way analysis of variance was conducted on the occipital $\mathrm{O} 2$ electrode, and the result is $p<0.05$. In addition, multiple comparisons show a significant difference between healthy subjects and those with MCI $(p<0.05)$ and those with MCI and those with $\mathrm{AD}(p<$ $0.10)$. The results of the occipital $\mathrm{O} 2$ electrode are described in Fig. 3 (wherein non-contributory (NC) refers to the healthy subjects).

In Fig. 3, it can be seen that subjects with MCI were attenuated compared with healthy subjects and those with AD. The same tendency was seen at other electrode sites. It is also clear that the data from the healthy subjects and those with MCI supports our hypothesis. However, the data from subjects with MCI and AD does not support our hypothesis. The same can be said about the overall average in Table 1.

Table 1 : Mean of $\beta / \alpha$ for $\mathrm{NC}$ and patients with MCI and AD

\begin{tabular}{|c|c|c|c|}
\hline $\begin{array}{c}\text { Electrode } \\
\text { site }\end{array}$ & $\begin{array}{c}\text { Healthy } \\
\text { subject }\end{array}$ & MCI & AD \\
\hline $\mathrm{Fz}$ & 0.91 & 0.86 & 1.12 \\
\hline $\mathrm{Cz}$ & 1.00 & 0.85 & 1.02 \\
\hline $\mathrm{P} 3$ & 0.91 & 0.86 & 1.01 \\
\hline $\mathrm{Pz}$ & 0.82 & 0.83 & 1.02 \\
\hline $\mathrm{P} 4$ & 1.13 & 1.03 & 1.24 \\
\hline $\mathrm{O} 1$ & 1.11 & 0.97 & 1.03 \\
\hline $\mathrm{Oz}$ & 1.20 & 1.03 & 1.13 \\
\hline $\mathrm{O} 2$ & 1.53 & 1.16 & 1.32 \\
\hline $\begin{array}{c}\text { Overall } \\
\text { average }\end{array}$ & 1.08 & 0.95 & 1.11 \\
\hline
\end{tabular}

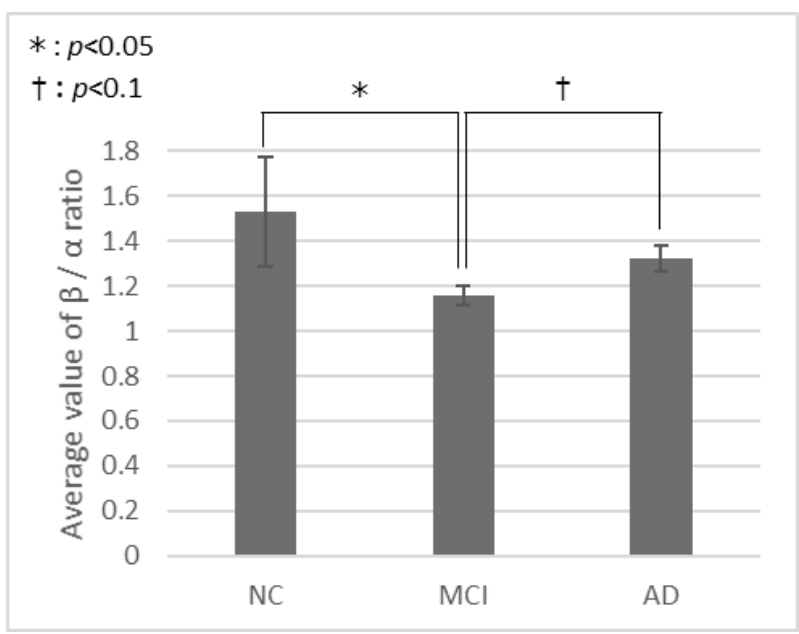

Figure 3: Mean of $\beta / \alpha$ for $N C$ and patients with $\mathrm{MCI}$ and $\mathrm{AD}$ (mean $\pm \mathrm{SD}, \mathrm{N}=56$ )

\section{Discussions}

With regard to the healthy subjects in Table 1 , the $\beta / \alpha$ mean value is generally $\geq 1$. Especially at the electrode sites of the occipital lobe $(\mathrm{O} 1, \mathrm{Oz}, \mathrm{O} 2)$, the $\beta / \alpha$ mean value is $>1$. This indicates $\beta$-wave dominance. With regard to patients with $\mathrm{MCI}$, it can be seen that the mean value of $\beta / \alpha$ tends to be $\leq 1$. This indicates $\alpha$-wave dominance. Based on this data, when considering the transition of subjects from being healthy to suffering from MCI, $\beta / \alpha$ can be considered an index of concentration; thus, it is highly possible that a decrease in a person's degree of concentration can be measured.

Next, regarding subjects with AD in Table 1, it can be seen that the mean value of $\beta / \alpha$ is $>1$ at all electrode sites; $\beta$ waves are considered superior to $\alpha$ waves. However, it is common knowledge that decreased $\alpha$ waves are a characteristic of EEGs of patients with dementia. Therefore, in this study, we also investigated the rate of $\theta$-wave generation. The analysis results are shown in Table 2 and Fig. 4. In Table 2, the data is summarized similar to that in Table 1 . As a result of multiple comparisons of the $\mathrm{Cz}$ electrode, a significant difference was observed between healthy subjects and those with MCI $(p<0.10)$ and subjects with MCI and those with AD $(p<0.05)$. These results are shown in Fig. 4 , the summary of which is the same as that of Fig. 3. From Fig. 4, it can be seen that the rate of $\theta$-wave generation in subjects with $\mathrm{AD}$ is increased compared with that of healthy subjects and patients with MCI. As such, it can be said that the reason the $\beta / \alpha$ mean value associated with subjects with $\mathrm{AD}$ is $>1$ is that the $\alpha$ wave is slowed down rather than the dominance of the $\beta$ wave.

Based on the above findings, it could be possible to 
Table 2: Mean of $\theta$ wave for $\mathrm{NC}$ and patients with $\mathrm{MCI}$ and $\mathrm{AD}$ $(\mathrm{N}=56)$

\begin{tabular}{|c|c|c|c|}
\hline $\begin{array}{c}\text { Electrode } \\
\text { site }\end{array}$ & $\begin{array}{c}\text { Healthy } \\
\text { subject(\%) }\end{array}$ & $\mathrm{MCI}(\%)$ & $\mathrm{AD}(\%)$ \\
\hline $\mathrm{Fz}$ & 30.22 & 35.33 & 38.01 \\
\hline $\mathrm{Cz}$ & 23.62 & 30.78 & 35.42 \\
\hline $\mathrm{P} 3$ & 22.35 & 25.59 & 32.36 \\
\hline $\mathrm{Pz}$ & 38.71 & 32.57 & 35.06 \\
\hline $\mathrm{P} 4$ & 21.67 & 27.09 & 30.46 \\
\hline $\mathrm{O} 1$ & 19.02 & 24.30 & 34.00 \\
\hline $\mathrm{Oz}$ & 26.60 & 28.66 & 31.41 \\
\hline $\mathrm{O} 2$ & 25.02 & 27.24 & 30.68 \\
\hline $\begin{array}{c}\text { Overall } \\
\text { average }\end{array}$ & 25.90 & 28.94 & 33.42 \\
\hline
\end{tabular}

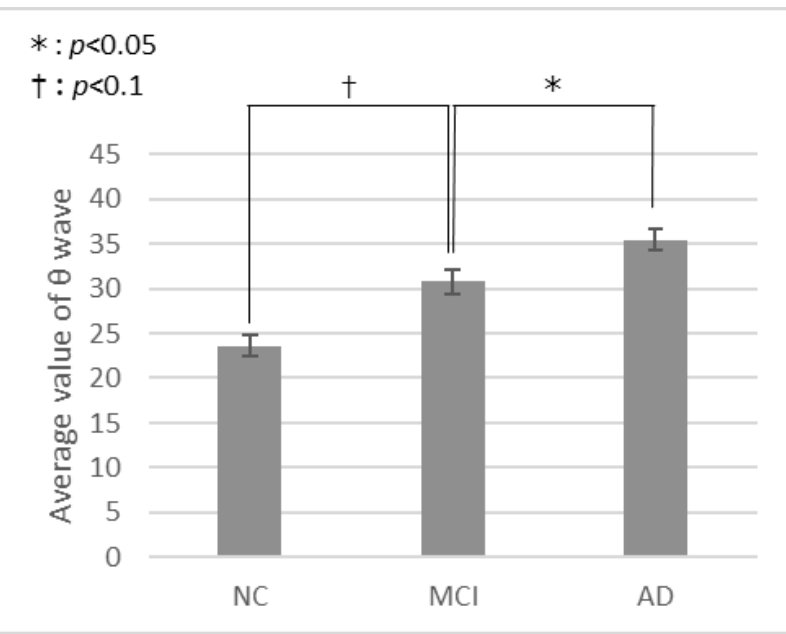

Figure 4: Mean of $\theta$ wave for $\mathrm{NC}$ and subjects with $\mathrm{MCI}$ and

$$
\mathrm{AD}(\text { mean } \pm \mathrm{SD}, \mathrm{N}=56)
$$

classify healthy subjects, those with MCI, and those with $\mathrm{AD}$ using the indices of $\beta / \alpha$ and $\theta$ waves. In addition, the purpose of clarifying the difference between healthy subjects and those in the pre-dementia stage was achieved because significant differences were found as a result of analysis using these indicators.

\section{Conclusion}

In this study, to identify the difference between the EEGs of healthy subjects and those with MCI, we evaluated the EEG data obtained at various angles using character-input-type BCI. In particular, we introduced new indices and analyzed and verified said indices in the frequency band. The results show that there is a high possibility that differences will be observed between healthy subjects, those with MCI, and those AD when it comes to the mean value of $\beta / \alpha$ and the generation rate of $\theta$ waves. This is thought to be because $\beta / \alpha$ is an index of concentration associated with cognitive decline, and $\theta$ waves are characteristic of EEGs of patients with dementia. Therefore, the purpose of finding a significant difference between healthy subjects and those MCI was achieved.

\section{Acknowledgments}

We would like to express our sincere gratitude to all the members of the Department of Elderly Care, Tokyo Medical University, for their cooperation in advancing this research. We would also like to express our deep gratitude to all the collaborators who cooperated with the experiment. Part of this research was carried out with a research fund for the development of minimally invasive treatment and diagnostic equipment, which is a joint research project of Tokyo Medical University and Kogakuin University. In addition, part of this research was carried out with the research funding of JSPS KAKENHI Grant Number JP19K12880.

\section{References}

[1] Ministry of Health, Labor and Welfare; Comprehensive Strategy for Promotion of Dementia Measures (New Orange Plan), https://www.mhlw.go.jp/file/06-Seisakujouhou-12300 000-Roukenkyoku/kaitei_orangeplan.pdf.

[2] Black CM, Ambegaonkar BM, Pike J, Jones E, Husbands J, Khandker RK; The Diagnostic Pathway From Cognitive Impairment to Dementia in Japan: Quantification Using Real-World Data, Alzheimer disease and associated disorders, Vol.33, No.4, pp.346-353, 2019

[3] Kurihara R, Morooka R, Tanaka H et al..; A New Assessment Method for the Cognitive Function in Dementia using the Spelling-BCI, The Transactions of Human Interface Society, Vol.21, No.1, pp.21-30, 2019.

[4] Mori A, Otomo E; Analysis of dementia by EEG, Japanese journal of cognitive neuroscience, Vol.3, No.1, pp.45-48, 2001

[5] Hirai F, Yoshida K, Miyaji I.; Comparison Analysis of the Thought and the Memory at the Learning Time by the Simple Electroencephalograph, Transaction of Multimedia, Distributed, Cooperative, and Mobile Symposium, pp.1441-1446, 2013.

[6] Haranaka Y, Ishida Y, Kurihara R; An Example of EEG Activity of the Prefrontal Cortex during Circuit Driving, Transactions of the Society of Automotive Engineers of Japan, Vol.41, No.2, pp.551-557, 2010. 